\title{
Up close and personal with Covid-19: An interview with Dr Usha Sriram
}

\author{
VIJAYAPRASAD GOPICHANDRAN
}

Keywords: Covid 19, pandemic, public health ethics, privacy, confidentiality

\section{Introduction}

The ongoing Covid-19 pandemic, starting in China in late 2019, has spread to every corner of the world, and thrown up several important ethical challenges. The rising numbers of infected persons and of death rates are keeping the health systems of most countries on their toes. However, the heightened focus on infection prevention and control have left several aspects of peoples' social life unaddressed. The stringent lockdowns in many countries including India, the mandatory public health measures, such as quarantine, isolation and contact tracing, have left a deep impact on the lives of the people.

Dr Usha Sriram is a senior consultant endocrinologist, practising in Chennai. The founder-director of DIWAS, a nonprofit organisation dedicated to the health of girls and women, she has pioneered the specialty of Endocrinology, Diabetes and Metabolism. Dr Usha continued to deliver clinical services at the TAG-VHS centre for diabetes and endocrinology at the peak of the Covid-19 pandemic. She and her elderly parents were diagnosed with Covid-19. In this interview with $\mathrm{Dr}$ Vijayaprasad Gopichandran, a Working Editor of IJME, Dr Usha shares her experiences of being diagnosed with Covid-19 from her varied perspectives as a physician, patient, care giver and member of the community. The interview was conducted on September 3, 2020.

Vijay: Hello, Dr Usha, it is heartening to have this conversation with you after your own and your family's complete recovery from Covid-19! From having the suspicion that you and your family could have been infected, through being tested, isolated, treated, followed up and finally released from your isolation today, the past couple of weeks must have been the experience of a lifetime. How are you now? What are your feelings about the past two weeks?

Dr Usha: Thanks, Vijay, for inviting me to do this interview about my experience with Covid 19. It has been a difficult two weeks and a strange mix of fear, confusion, uncertainty, and a sense of panic that I have never felt before. Our family has had

Author: Vijayaprasad Gopichandran (vijay.gopichandran@gmail.com), Assistant Professor, Department of Community Medicine, ESIC Medical College and PGIMSR, KK Nagar, Chennai 600078, INDIA.

To cite: Gopichandran V. Up close and personal with Covid-19: An interview with Dr Usha Sriram. Indian J Med Ethics. 2021 Apr-Jun; 6(2) NS: 140-142. DOI: 10.20529/IJME.2020.132.

Published online first on December 24, 2020.

(c) Indian Journal of Medical Ethics 2020 its share of encounters with health issues from heart problems, fractures, and gastro-intestinal bleeds to swine flu, but never has there been such uncertainty and such conflict in the approach to treatment.

This experience has been an eye opener in so many ways. I have a heightened sense of what it feels to be a care-giver, a healthcare provider within the health system and a member of the community dealing with the public health system during these pandemic times.

Vijay: Let us start from the point when you got the positive diagnosis of Covid-19. Could you please explain how the public health system responded to your diagnosis? What were the immediate actions taken?

Dr Usha: The diagnosis of Covid-19 was immediately accompanied by disbelief followed by the Chennai Municipal Corporation officials coming and affixing the sticker indicating that our home has Covid 19 infected individuals, barricading it to prevent the general public from accidentally entering, and Corporation officials visiting us to organise our transport to the relevant Covid-19 treatment facilities.

The personnel from the Chennai Corporation visited us about 48 hours after the test results of my dad, who was the first one with symptoms in our family and wanted to take him to a nearby Covid-19 Care Centre. When I explained that he is 91 years of age and the family and his physician have decided to isolate and treat him at home, they wanted a letter from the doctor stating all that. And they wanted it within the next couple of hours. I had to immediately make phone calls to our family physicians to arrange for this letter. They then took the letters from all four of us who tested positive, sprayed our home with sanitisers and then left.

Vijay: These interventions seem quite rigorous and thorough, though a bit delayed. Did you experience any burdens, difficulties, or problems because of them?

Dr Usha: Because I am a physician myself, and I understand the protocols and procedures to an extent, and I could get a quick evaluation as well as the letters from my physician, it was not particularly difficult. But the clock was ticking, and organising everything within the tight timeline was stressful.

They calculated the period of 14 days of isolation from the date of their visit to our home, which itself was 48 hours after we were diagnosed. It should have been 14 days from the date of diagnosis.

I suffer from a hyperactive airway and develop wheezing and cough when I encounter any incense, spray, or dust. So, I was apprehensive about the sanitiser spraying operation in our home. My dad also suffers from emphysema, a lung condition that leaves him over-sensitive to such fumes. So, I had to insist 
that they do the sanitiser spraying operation only outside the home.

I cannot help but wonder about people who are not physicians and who do not have the support system to understand and negotiate these issues. One of the important points I noticed was that the people who landed at my doorstep to implement the public health actions were not public health professionals or community health workers but Covid-19 volunteers with varying levels of training and understanding about the pandemic. The persons who came to my home for the response lacked the sensitivity and compassion that is required in a frontline health worker, especially when interacting with a family affected by Covid19. While I respect their commitment to the risky work that these volunteers and corporation officials did for us, I think it would have been better to have sensitive and compassionate workers in the frontline.

Vijay: I am so sorry to hear about that. Do you think this could have been avoided?

Dr Usha: Yes, for many people in the community, the problem is that they don't know what to expect once they are diagnosed with Covid-19. There is a lot of emphasis on the public announcements about preventive measures against contracting the infection such as wearing masks, avoiding crowded spaces, maintaining social distancing etc. But what can one expect from the Corporation once they are diagnosed with Covid-19? This is not clear. Probably a detailed description of what to expect through TV, print media, radio, posters, and flyers would have mentally prepared me for the public health interventions. I would not call any of the interventions that the Corporation carried out unreasonable. They were all important. But I would have been better prepared had I known that the corporation officials would make a home visit after the diagnosis and would do all that they did.

Vijay: That is a very good suggestion. The public health system can actively disseminate information on what to expect after a diagnosis of Covid-19 is made. One other public health intervention that is actively undertaken during such pandemics is contact tracing. Did the public health system trace your contacts? How did they do this?

Dr Usha: I was surprised and upset when several friends and colleagues started calling me about phone calls, they had been receiving from the Corporation and the police. The public health system had tracked all my phone contacts with whom I had talked in the few days preceding my diagnosis. This was not something that I was aware of. While I understand that these are unusual and difficult times for public health authorities, it was a definite invasion of privacy. The public health officials called my contacts and informed them about my diagnosis of Covid-19 and asked them to quarantine themselves if they have been in contact with me. Many of these contacts are purely telephonic contacts. I had not even met them. So, I think tracking all telephonic contacts is not a particularly good idea for contact tracing. Firstly, I think this is a violation of my privacy. However, I do understand these are difficult times and I am willing to cooperate, but I should have at least been informed that all my telephonic contacts will be traced and informed of my Covid-19 diagnosis. There was no attempt made to talk to me and ask me about all my contacts. Without attempting the traditional contact tracing method with me, in which case I would have fully disclosed all my contacts, invading my privacy through tracing my phone calls was unreasonable

Vijay: I am sorry to hear about your experience with contact tracing. How did the public health system respond with respect to follow-up during your treatment?

Dr Usha: Since all four of us in my family were treated from home, we received phone calls from the Corporation personnel every day. I had given my phone number for all four of us and so I would receive four phone calls a day, one for follow up of each one of us. I received these phone calls every single day for the 14 days of isolation. Each call would last roughly 5-7 minutes. They would ask us how we are doing, ask us in detail about our symptoms, check if we are improving, and advise us on what to eat, what medicines to take etc. The personnel who called were very polite and thorough. They must have a standard text to read out and a checklist to fill out. They did their job diligently. But imagine listening to the same thing 56 times over 14 days. Attending and responding to the phone calls itself drained a lot of my energy. Add to this phone calls of friends, family, people who were contacted by the public health officials as part of the contact tracing exercises, and the phone was constantly ringing.

It would have been much better if there was continuity of the person who called to check in on our family. Different people called on each of the 14 days and it was a fresh new interview each time. This is not helpful. For example, the person who called asked us to consume plenty of fluids of about 8-10 glasses per day. My father has a heart condition because of which we restrict the fluid intake. But the different persons on the calls each day kept repeating this instruction. The first couple of days I explained to the person on the call about the fluid restriction, but then I just started saying OK to everything they said. I am a physician, and I could navigate this with minimum trouble, but I cannot imagine the plight of the lay public, who must be getting conflicting messages through these phone calls. If there was one caseworker for a person or a family, it would have been much easier, and would have helped establish rapport and continuity of care.

Vijay: The telephone calls sound like a difficult chore. Do you think the invasion of privacy, these cumbersome phone calls every day and the other public health measures will have an impact on people's response to the pandemic?

Dr Usha: Definitely, all these actions will push people away from the public health system. I already see many people hiding from the public health system not revealing their symptoms and not seeking healthcare out of fear of all these procedures. The health system must think of how they can make these interventions more people-friendly so as not to chase them away into hiding.

Vijay: You are in a unique position as a physician, a family member, a care giver and a patient, all at the same time. How difficult was it to make clinical decisions about the treatment of Covid-19 for yourself and your family?

Dr Usha: While I am fortunate enough to have extraordinarily talented and helpful physician colleagues, each one seasoned, competent, and very well respected, you couldn't find more a more diverse set of assessments and recommendations. From "do nothing" to "let us try the entire menu available" including Dexamethasone, Remdesivir, Convalescent Plasma, Antibiotics and everything in between. One half of my brain definitely liked the "do nothing" approach especially because it came from a couple of highly regarded and brilliant clinicians, while 
the other half of my brain started wondering if I was doing everything I should be doing, especially for my elderly parents. It was undeniably a difficult time dealing with these conflicts. I went with the "less is more approach". While I think this might not be the case for the public, I can empathise with the clinicians who are treating patients with Covid-19, and the uncertainty and dilemmas that they must be going through.

Another interesting phenomenon that seems to be unique to this pandemic is that everyone seems be a Covid-19 expert! Several non-physician cousins of mine were forwarding contact details of Siddha medicine specialists and suggesting other immune-boosting therapy ideas. They were comfortable talking about pulse oximetry and one person asked what the CT scan showed!

And there are others who believe that a lot of the Covid-19 news is fake news and a hoax. Many educated and aware people firmly subscribe to these views. They are convinced that labs, hospitals and the state governments and the media are exaggerating the crisis into something much bigger for financial gain and sensationalism.

It can be a challenge to navigate all this with a calm and positive disposition.

Vijay: What messages do you have from these experiences about the ethics of pandemic response?

Dr Usha: Fundamentally there needs to be de-stigmatising of the Covid-19 positive status. Stickers on doors, barricades, involvement of the police department, a "one size fits all" approach, and non-healthcare personnel involved in the process, all make for an experience that is far from satisfactory. A general sense of being victimised starts from the first encounter. All this can be avoided with increasing awareness, greater emphasis on the persons rather than the disease, and more sensitive handling of the public health interventions.

Vijay: How should the ethical conduct of public health interventions during pandemic times be monitored?

Dr Usha: I think we should have regular updates from the public health authorities on:

- what to expect when you test positive;

- what to expect in the hospital including cost;

- who will apprise the family regularly of the patient's wellbeing;

- what asymptomatic Covid-19 positive people should expect and what they should do.

While the government machinery for information dissemination is doing a fantastic job of spreading messages on how to prevent Covid-19, there is a dearth of focus on the above issues. The only sources of information people have are the media and social media and those are riddled with myths, misconceptions, finger-pointing and politicisation.

Vijay: Thank you very much for sharing your experiences with Covid-19. Your personal insights into the pandemic and the public health interventions throw light on the important and often neglected ethical issues related to public health interventions during pandemic times.

\section{Discussion}

Public health emergencies and pandemics do not offer excuses to health systems to take the lives of people and communities for granted. While it is a key imperative for health systems to control infection and mitigate the damage caused by such pandemics, the health system must closely consider how its interventions will impact the social lives of people.

Often public health interventions during pandemic times impinge on individual rights and liberties. The restrictive public health measures of isolation and quarantine grossly limit the movements of people and their freedom. The strict lockdown imposed by the government not only restricted the liberty of people, but stripped many of their livelihood. The economy of the country is facing a major slump and it has its own negative impact on the health and wellbeing of people. While these public health interventions are necessary to contain the spread of the pandemic, they also lead to serious hardships and difficulties. The balance between the benefits of these public health measures and the risks that they carry is often delicate and difficult to achieve.

Contact tracing, name-based reporting of people diagnosed with Covid-19, public announcement of Covid-19 positive status by putting up stickers on doors of homes, all breach the privacy and confidentiality of individuals. This subjects them to stigmatisation. Here again, the balance between performing these key public health activities all the while respecting the individual who stands to lose because of these interventions is difficult to achieve.

There is a need to evolve public health strategies with a tinge of sensitivity to the individuals and communities who are at the centre of the pandemic. Community engagement, informing them adequately that there are going to be restrictions of movement, allowing them adequate time to prepare for the restrictions, putting social welfare measures in place to compensate for the restrictions are all sensitive interventions which can make the public health measures more ethical. Informing communities that contact tracing will be done, and it is likely to violate their individual privacy can help them be prepared

Another important ethical consideration during this pandemic is the uncertainty about treatments and treatment decisions. Innumerable unproven therapies, experimental treatments, repurposed drugs are being tried among patients. Rapid and inaccurate research is conducted, often not giving rise to conclusive evidence for or against treatments. This leads to substantial confusion about the appropriate treatment for patients diagnosed with Covid-19. In addition, a myriad complementary and alternative therapies are also advocated actively by the government as well as individuals. The scientific basis behind all these unproven remedies is a matter of serious ethical concern.

From this interview it is clear there is a need to focus on the ethics of public health interventions. Public health systems must incorporate ethics reviews in all their interventions in order to keep even the most restrictive ones people-centred and sensitive. 